\title{
From LO to NLO in the parton Reggeization approach
}

\author{
Maxim Nefedov ${ }^{1,2, *}$ and Vladimir Saleev ${ }^{1, * *}$ \\ ${ }^{1}$ Samara National Research University, Samara, Russia \\ ${ }^{2}$ Hamburg University, Hamburg, Germany
}

\begin{abstract}
We present recent developments of the parton Reggeization approach (PRA), which is based on high-energy factorization of hard processes in the multi-Regge kinematics and Lipatov's effective theory of Reggeized gluons and Reggeized quarks. The scheme of calculations in the leading order (LO) of the PRA is discussed. We present important examples of LO PRA applications for cross section calculations of multi-scale hard processes, such as pair production of $B \bar{B}$-mesons, pair production of photons, and pair production of jets. Also the problem of matching of NLO calculations in PRA with NLO results in the Collinear Parton Model is discussed.
\end{abstract}

\section{Introduction}

To describe high-energy hadron-hadron or lepton-hadron collisions in Quantum Chromodynamics (QCD), one usually have to introduce some kind of factorization, which separates the effects of long and short distances [1,2]. Appropriate factorization formula should be chosen accordingly to the kinematics of the observable under consideration. Factorization in the conventional collinear parton model (CPM) is usually applied in case of the single-scale hard processes [2]:

$$
\sigma^{\mathrm{CPM}}=\sum_{q, g} \int d x_{1} \int d x_{2} f_{q, g}\left(x_{1}, \mu\right) f_{q, g}\left(x_{2}, \mu\right) \hat{\sigma}_{\mathrm{CPM}}\left(x_{1}, x_{2}, \mu\right)+O\left(\Lambda^{2} / \mu^{2}\right),
$$

where $f_{q, g}(x, \mu)$ are collinear parton distribution functions (PDFs), $\mu$ is the scale of hard process, $\hat{\sigma}_{\mathrm{CPM}}$ is the cross section of quark-gluon interaction, and $\Lambda^{2}$ is the typical energy scale of non-perturbative phenomena in QCD. The collinear PDFs satisfy perturbative DGLAP evolution equations [3], which resum contributions of large logarithms $\sim \log \left(\mu^{2} / \Lambda^{2}\right)$. Partonic cross section $\hat{\sigma}$ is calculated order by order of perturbative QCD expansion for on-shell collinear partons, carrying the fractions $x_{1,2}$ of momentum of initial-state protons and no transverse momentum.

In the so-called transverse momentum depended (TMD) factorization scheme [2], factorization formula of CPM (1) is generalized by inclusion of small transverse momenta of initial partons,

$$
\begin{aligned}
\sigma^{\mathrm{TMD}} & =\sum_{q, g} \int d x_{1} \int d \mathbf{q}_{1 T} \int d x_{2} \int d \mathbf{q}_{2 T} F_{q, g}\left(x_{1}, \mathbf{q}_{1 T}, \mu\right) F_{q, g}\left(x_{2}, \mathbf{q}_{2 T}, \mu\right) \times \\
& \times \hat{\sigma}_{\mathrm{CPM}}\left(x_{1}, x_{2}, \mu\right) \delta^{(2)}\left(\mathbf{q}_{1 T}+\mathbf{q}_{2 T}-\mathbf{p}_{T}\right)+O\left(\Lambda^{2} / \mu^{2}, \mathbf{p}_{T}^{2} / \mu^{2}\right),
\end{aligned}
$$

\footnotetext{
*e-mail: manefedov@gmail.com

**e-mail: saleev@samsu.ru
} 
where $F_{q, g}\left(x, \mathbf{q}_{T}, \mu\right)$ are TMD PDFs [4], $\mathbf{p}_{T}$ is the transverse momentum of final particles in the hard scattering, $\hat{\sigma}_{\mathrm{CPM}}$ is the cross section of hard parton-parton scattering, obtained in the collinear approximation. In the TMD factorization approach we can include nonperturbative effects of initial transverse momenta, which are needed to describe data at small transverse momenta, $\left|\mathbf{p}_{T}\right| \ll \mu$, because large logarithmic contributions enhanced by $\log \left(\mu^{2} / \Lambda^{2}\right)$ and $\log ^{n}\left(\mu^{2} / \mathbf{p}_{T}^{2}\right)(n=1,2)$ are taken into account in the TMD PDFs [5].

At very high energy, when $\mu / \sqrt{S} \ll 1$, the high-energy or $k_{T}$-factorization corresponds to the assumption of domination of $t$-channel diagrams with all final-state particles highly separated in rapidity in the high-energy asymptotics of any multi-particle production amplitude (so-called Multi-Regge Kinematics, MRK). The $k_{T}$-factorization formula reads

$$
\begin{aligned}
\sigma^{\mathrm{KT}} & =\int d x_{1} \int d \mathbf{q}_{1 T} \int d x_{2} \int d \mathbf{q}_{2 T} \Phi_{g}\left(x_{1}, \mathbf{q}_{1 T}\right) \Phi_{g}\left(x_{2}, \mathbf{q}_{2 T}\right) \times \\
& \times \hat{\sigma}_{\mathrm{KT}}\left(x_{1}, x_{2}, \mathbf{q}_{1 T}, \mathbf{q}_{2 T}, \mu\right)+O\left(\Lambda^{2} / \mu^{2}, \mu^{2} / S\right),
\end{aligned}
$$

where $\Phi_{q, g}\left(x, \mathbf{q}_{T}\right)$ are "unintergrated" PDF (unPDF) for off-shell (Reggeized) gluon in $t$ channel with virtuality $q^{2}=-\mathbf{q}_{T}^{2}<0$, while the hard scale $\mu \sim\left|\mathbf{p}_{T}\right|$. Unintegrated PDF is subject of asymptotic BFKL evolution equation [6] which resums large logarithmic contributions $\sim \log \left(S / \mu^{2}\right)$. Partonic cross section, $\hat{\sigma}_{\mathrm{KT}}$ for off-shell initial gluons is calculated as multi-Regge limit of relevant QCD amplitudes with two additional final gluons or quarks [7].

However, there are many multi-scale observables in high-energy collisions lacking clear separation of involved scales, which would make one of above-mentioned factorization formulas applicable. The object of our special concern are the azimuthal decorrelation observables in the whole range of $0 \leq \Delta \phi \leq \pi$, which can be studied in the pair production of jets, photons, mesons e.t.c. In principle, the fully-unintegrated factorization, which takes into account all four components of momentum of initial-state parton is needed to describe such processes $[2,8]$, however at present there is no systematic theory of such factorization and it is not clear neither how to calculate the gauge-invariant hard scattering coefficient (HSC) for the arbitrary process ${ }^{1}$ nor what kind of evolution equation governs the fully-unintegrated PDFs.

Parton Reggeization Approach is an attempt to extend the formalism of High-Energy factorization to deal with above-mentioned multi-scale observables without introduction of fully-unintegrated PDF. The brief description of LO factorization in PRA is presented below. More details can be found in [9], the development of PRA in the NLO approximation is further discussed in $[10,11]$.

\section{The LO framework of PRA}

In this part, we discuss the structure of LO calculations in PRA. The main ingredients of PRA are factorization formula, unintegrated parton distribution functions (unPDF's) and Lipatov's Effective Field Theory (EFT) of Reggeized gluons [12] and Reggeized quarks [13].

The systematic formalism for the calculation of the asymptotic expressions for arbitrary QCD amplitudes in the MRK limit has been formulated by L. N. Lipatov and M. I. Vyazovsky in a form of gauge-invariant Effective Field Theory (EFT) for Multi-Regge processes in QCD [12, 13], see also [14] for a review. The MRK asymptotics of the amplitude in this EFT is constructed from gauge-invariant blocks - effective vertices, which describe the production of clusters of QCD partons, separated by the large rapidity gaps. These effective vertices are connected together via $t$-channel exchanges of gauge-invariant off-shell degrees of freedom: Reggeized gluons $R_{ \pm}$and Reggeized quarks $Q_{ \pm}$.

\footnotetext{
${ }^{1}$ See however [15] for the example of definition of fully-unintegrated NLO HSC for the Deep Inelastic Scattering.
} 
Due to the requirements of gauge-invariance of effective vertices, the interactions of QCD partons and Reggeons in the EFT [12,13] are nonlocal and contain the Wilson's exponents of gluonic fields. After perturbative expansion, the latter generate an infinite series of induced vertices of interaction of particles and Reggeons. The Feynman Rules of Lipatovs EFT, are worked out in details in the Ref. [16].

Factorization formula of PRA in LO approximation for the process $p+p \rightarrow \mathcal{Y}+X$, can be obtained from factorization formula of the collinear parton model (CPM) for the auxiliary hard subprocess $g+g \rightarrow g+\mathcal{Y}+g$. In the Ref. [9] the modified MRK (mMRK) approximation for this amplitude is constructed (see the diagrammatic representation of it in the left panel of Fig. 1), which correctly reproduces the MRK and collinear limits of corresponding QCD amplitude.

This mMRK-amplitude has $t$-channel factorized form, which allows one to rewrite the cross-section of auxiliary subprocess in a $k_{T}$-factorized form:

$$
d \sigma=\int_{0}^{1} \frac{d x_{1}}{x_{1}} \int \frac{d^{2} \mathbf{q}_{T 1}}{\pi} \tilde{\Phi}_{g}\left(x_{1}, t_{1}, \mu^{2}\right) \int_{0}^{1} \frac{d x_{2}}{x_{2}} \int \frac{d^{2} \mathbf{q}_{T 2}}{\pi} \tilde{\Phi}_{g}\left(x_{2}, t_{2}, \mu^{2}\right) \cdot d \hat{\sigma}_{\mathrm{PRA}},
$$

where $t_{1,2}=-\mathbf{q}_{T 1,2}^{2}$, the partonic cross-section $\hat{\sigma}_{\mathrm{PRA}}$ in PRA is determined by squared PRA amplitude, $\overline{\left|\mathcal{A}_{P R A}\right|^{2}}$, and the tree-level "unPDFs" $\tilde{\Phi}_{g}\left(x_{1,2}, t_{1,2}, \mu^{2}\right)$ are equal to the convolution of the relevant collinear PDF $f_{g}\left(x, \mu^{2}\right)$ and DGLAP splitting function $P_{g g}(z)$ :

$$
\tilde{\Phi}_{g}\left(x, t, \mu^{2}\right)=\frac{1}{t} \frac{\alpha_{s}}{2 \pi} \int_{x}^{1} d z P_{g g}(z) \cdot \frac{x}{z} f_{g}\left(\frac{x}{z}, \mu^{2}\right) .
$$

The squared PRA amplitude is defined as:

$$
\overline{\left|\mathcal{A}_{P R A}\right|^{2}}=\left(\frac{q_{1}^{+} q_{2}^{-}}{4\left(N_{c}^{2}-1\right) \sqrt{t_{1} t_{2}}}\right)^{2}\left[\mathcal{A}_{c_{1} c_{2}}^{\star} \mathcal{A}^{c_{1} c_{2}}\right],
$$

where $\mathcal{A}$ is the Green's function of the subprocess $R_{+}\left(q_{1}\right)+R_{-}\left(q_{2}\right) \rightarrow \mathcal{Y}\left(P_{\mathcal{A}}\right)$ in Lipatov's EFT [12] with amputated propagators of the Reggeized gluons $\left(R_{ \pm}\right)$, and $c_{1,2}$ are their color indices.

The cross-section (4) with "unPDFs" (5) contains the collinear divergence at $t_{1,2} \rightarrow 0$ and infrared (IR) divergence at $z_{1,2} \rightarrow 1$. To regularize the latter, we observe, that the mMRK expression can be expected to give a reasonable approximation for the exact matrix element only in the rapidity-ordered part of the phase-space, where $\Delta y_{1}>0$ and $\Delta y_{2}>0$. From this requirement, the following cutoff on $z_{1,2}$ can be derived: $z_{1,2}<1-\Delta_{K M R}\left(t_{1,2}, \mu^{2}\right)$, where $\Delta_{K M R}\left(t, \mu^{2}\right)=\sqrt{t} /\left(\sqrt{\mu^{2}}+\sqrt{t}\right)$ is the KMR cutoff function $[8,18]$, and we have taken into account that $\mu^{2} \sim M_{T \mathcal{A}}^{2}$. The collinear singularity is regularized by the Sudakov formfactor:

$$
T_{i}\left(t, \mu^{2}\right)=\exp \left[-\int_{t}^{\mu^{2}} \frac{d t^{\prime}}{t^{\prime}} \frac{\alpha_{s}\left(t^{\prime}\right)}{2 \pi} \sum_{j=q, \bar{q}, g} \int_{0}^{1} d z z \cdot P_{j i}(z) \theta\left(1-\Delta_{K M R}\left(t^{\prime}, \mu^{2}\right)-z\right)\right],
$$

which resums doubly-logarithmic corrections $\sim \log ^{2}\left(t / \mu^{2}\right)$ in the LLA in a way similar to what is done in the standard parton-showers [17].

The final form of our unPDF in PRA is:

$$
\Phi_{i}\left(x, t, \mu^{2}\right)=\frac{T_{i}\left(t, \mu^{2}\right)}{t} \frac{\alpha_{s}(t)}{2 \pi} \sum_{j=q, \bar{q}, g} \int_{x}^{1} d z P_{i j}(z) \cdot \frac{x}{z} f_{j}\left(\frac{x}{z}, t\right) \cdot \theta\left(1-\Delta_{K M R}\left(t, \mu^{2}\right)-z\right),
$$



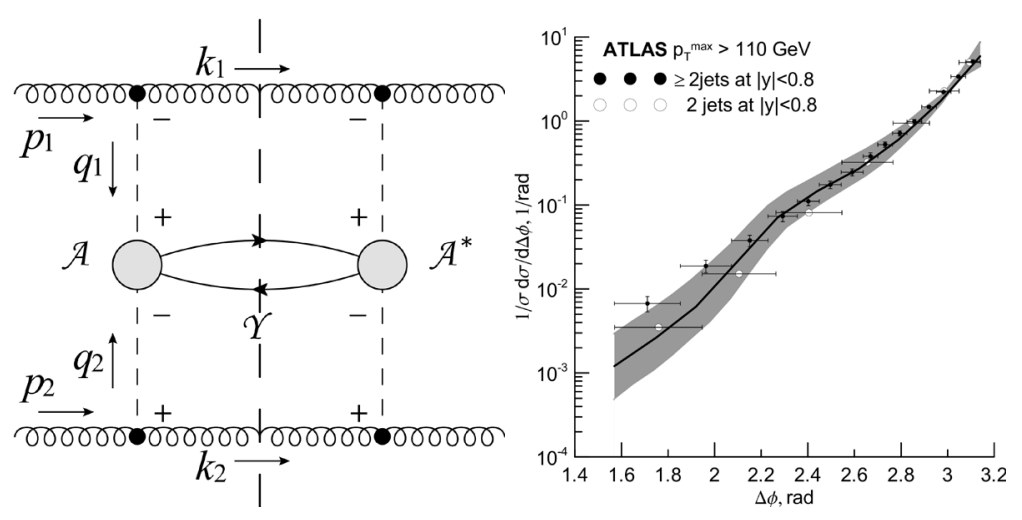

Figure 1. Left panel: diagrammatic representation of the MRK asymptotics for squared amplitude of the auxiliary hard subprocess. Right panel: normalized $\Delta \phi$-distribution for 2 (open circles) and $\geq 2$ (black circles) jets with $p_{T}>100 \mathrm{GeV},|y|<0.8, p_{T}>110 \mathrm{GeV}$ and $\sqrt{S}=7 \mathrm{TeV}$. The data are from the ATLAS Collaboration [27].

which coincides with Kimber, Martin and Ryskin (KMR) unPDF [18]. The KMR unPDF is actively used in the phenomenological studies employing $k_{T}$-factorization, but to our knowledge, the derivation, presented in [9] is the first systematic attempt to clarify it's relationships with MRK limit of the QCD amplitudes.

The main difference of PRA from the multitude of studies in the TMD-factorization and $k_{T}$-factorization is the application of matrix elements with off-shell initial-state partons (Reggeized quarks and gluons) from Lipatov's EFT [12,13], which allows one to study the arbitrary processes involving non-Abelian structure of QCD without violation of gaugeinvariance due to the nonzero virtuality of initial-state partons. This approach, together with KMR unPDF gives stable and consistent results in a wide range of phenomenological applications, which include the description of the angular correlations of dijets [19], $b$-jets [20], charmed [21, 22] and bottom-flavored [9, 23] mesons, different multiscale observables in hadroproduction of diphotons [24] and photoproduction of photon+jet pairs [25], as well as some other examples.

\section{Azimuthal decorrelations of dijets, diphotons and $B \bar{B}$-pairs}

The study of azimuthal angle difference spectra between two high- $p_{T}$ jets, two photons, or two heavy mesons is one of the most challenging observables in pQCD. Calculations in the CPM, sometimes even in NNLO approximation(e.g. for diphotons. See Ref. [26]), can not describe data in the region $\Delta \phi<\pi / 2$, and especially for small $\Delta \phi$. Inclusion of small nonperturbative transverse momenta of initial partons in the TMD-factorization approach is also relevant only for $\Delta \phi \simeq \pi$. In the PRA, initial transverse momenta of Reggeized partons are sufficiently large, that is why already in the LO PRA ( $2 \rightarrow 2$ subprocesses) one can describe most part of the cross-section at small $\Delta \phi$.

In the right panel of Fig. 1, our prediction for dijets azimuthal spectrum is compared with data from ATLAS Collaboration [27]. LO PRA calculation well describes the data for events with only two high- $p_{T}$ jets in the central region of rapidity. In case of events with more than two high- $p_{T}$ jets, the NLO corrections are needed.

Azimuthal decorrelations in production of pairs of isolated prompt photons has been studied both at Tevatron and LHC. In the Fig. 2, we compare our prediction, obtained in the PRA, 
with the CDF data [28]. We include LO contribution from $Q \bar{Q} \rightarrow \gamma \gamma$ subprocess, real NLO contribution from $Q R \rightarrow q \gamma \gamma$ subprocess and important part of NNLO contribution from $R R \rightarrow \gamma \gamma$ subprocess. Description of the data is rather satisfactory, excluding region of back-to-back production, where the cross-section is enhanced by virtual and soft-collinear corrections, absent in our calculations.

In CMS analysis of Angular correlations in pair production of $B \bar{B}$-mesons [29], production of high-energy (leading) jet is used as trigger of hard production of $B \bar{B}$-meson pair. If leading jet is $b(\bar{b})$-jet, the relevant LO partonic subprocess is $R R \rightarrow b \bar{b}$. To simulate events where the leading jet is not $b$-tagged, we use the merging technique [9], to consistently take into account the NLO contribution from subprocess $R R \rightarrow b \bar{b} g$ with the hard gluon initiating the leading jet. After including this NLO correction we have found very good agreement with data, see Fig. 3.

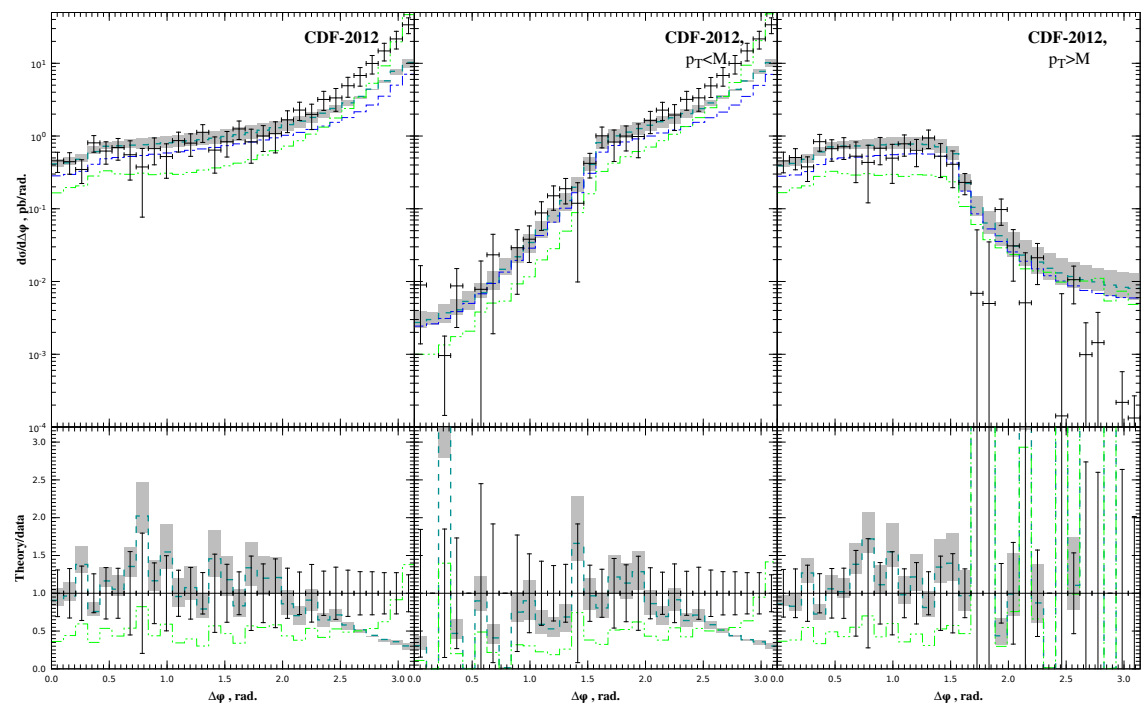

Figure 2. The $\Delta \phi$-spectra for the CDF dataset [28]. The thick dashed curve is the sum of the contributions $Q \bar{Q} \rightarrow \gamma \gamma, Q R \rightarrow q \gamma \gamma$, and $R R \rightarrow \gamma \gamma$. The thin dash-dotted curve is the contribution of the subprocess $Q \bar{Q} \rightarrow \gamma \gamma$ only. The thin dash-double-dotted curve is the corresponding Diphox (NLO CPM) prediction, taken from the Ref. [28].

\section{Towards NLO calculations in PRA}

The problem of NLO computations in PRA, mainly consists in formulating of the subtraction prescription for the real and virtual corrections to the HSC, which will ensure it's finiteness and absence of double-counting of radiative corrections, which already have been taken into account in the LO. The main guiding physical principle here is the physical normalization condition $(P N C)$, which states that NLO results for single-scale observables in PRA should coincide with corresponding results in CPM up to the terms of higher-order in $\alpha_{s}\left(\mu^{2}\right)$ and corrections power-suppressed by the hard scale $\mu^{2}$. In the LO, this condition is satisfied automatically because for the KMR unPDF (8) the following normalization condition holds:

$$
\int_{0}^{\mu^{2}} d t \Phi_{i}\left(x, t, \mu^{2}\right)=x f_{i}\left(x, \mu^{2}\right) .
$$



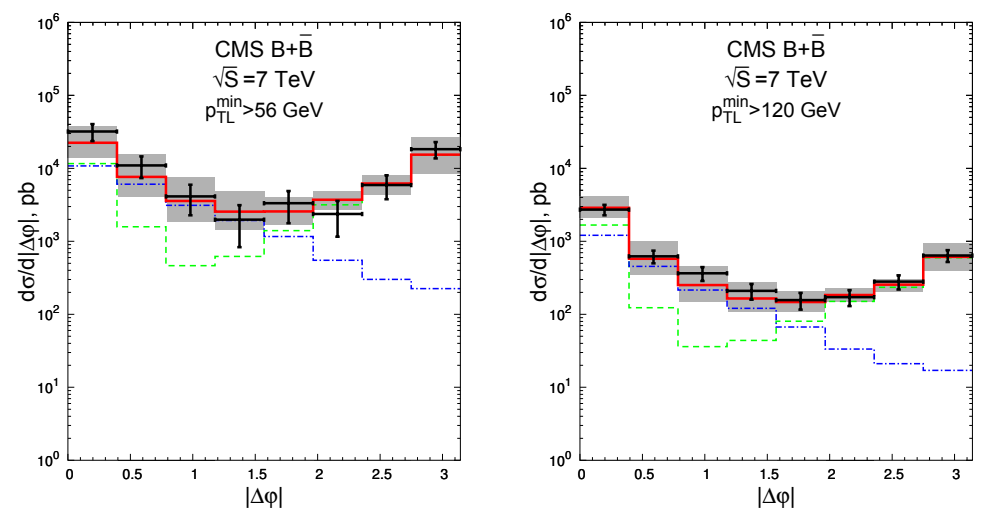

Figure 3. Comparison of the predictions for $\Delta \phi$-spectra of $B \bar{B}$-pairs with the CMS data [29]. Dashed line is contribution of the LO subprocess $(R R \rightarrow b \bar{b})$, dash-dotted line is contribution of the NLO subprocess $(R R \rightarrow b \bar{b} g)$, solid line is the sum of LO and NLO contributions.

The LO KMR unPDF in the form (8) satisfies Eq. (9) only at small- $x$. The unPDF, satisfying the condition (9) exactly can be constructed only by introducing a certain $x$-dependence to the Sudakov formfactor, See Eqns. 12 and 13 in Ref. [11].

One can choose any single-scale observable, to perform matching between PRA and CPM. In the Ref. [11] we study the simplest tree-level $O\left(\alpha_{s}\right)$ contribution to the structurefunction of Deep Inelastic Scattering on a certain quark flavour ${ }^{2} F_{2 q}\left(x_{B}, Q^{2}\right)$ (where $x_{B}=$ $Q^{2} /(2 P q)$ is the usual Bjorken variable):

$$
R_{+}\left(q_{1}\right)+\gamma^{\star}(q) \rightarrow q\left(k_{1}\right)+\bar{q}\left(k_{2}\right)
$$

In PRA, contribution of the subprocess (10) to the structure-function does not contain explicit collinear divergence, in contrast to CPM. Instead, the HSC contains $\log \left(t_{1} / Q^{2}\right)$, which yields a finite contribution upon integration with unPDF. But this collinear logarithm was already taken into account in our LO factorization formula. Another source of double-counting is MRK region, where the rapidity gap $\Delta y\left(k_{1}, k_{2}\right) \gg 1$. In the Ref. [11], contributions of both of this regions are subtracted on the level of integrand of the HSC by corresponding mMRK subtraction terms (See Eqns. 29, 30, 31 in Ref. [11]). After this subtraction, the HSC itself become finite in the limit $t_{1} \rightarrow 0$ and therefore, the $O\left(\alpha_{s}\right)$ contribution of the subprocess (10) is given by

$$
F_{2 q}^{(1, g)}\left(x_{B}, Q^{2}\right)=\frac{\alpha_{s}\left(Q^{2}\right)}{2 \pi}\left[C_{2}^{(1, g)} \otimes f_{g}\right]+O\left(\alpha_{s}^{2}\right)+O\left(\left(Q^{2}\right)^{-\gamma}\right)
$$

where $[C \otimes f]=\int_{x_{B}}^{1} \frac{d z}{z} C\left(x_{B} / z\right) f\left(z, Q^{2}\right)$ is the Mellin convolution, $C_{2}^{(1, g)}$ is the collinear limit of the subtracted PRA hard-scattering coefficient, $O\left(\alpha_{s}^{2}\right)$ and $O\left(\left(Q^{2}\right)^{-\gamma}\right)$ are respectively higher-order and higher-twist effects arising due to nontrivial dependence of NLO HSC in PRA on $t_{1}$.

The function $C_{2}^{(1, g)}$ is represented in Ref. [11] as:

$$
C_{2}^{(1, g)}(z, 0)=2\left[C_{2 g}^{\overline{M S}}(z)-\Delta C_{q g}(z)\right],
$$

\footnotetext{
${ }^{2}$ Which is a gauge-invariant, single-scale quantity well-defined in any order of QCD perturbation theory.
} 
where $C_{2 g}^{\overline{M S}}$ is the textbook HSC for the subprocess $g+\gamma^{\star} \rightarrow q+\bar{q}$ in CPM, computed in the $\overline{M S}$-scheme, and $\Delta C_{q g}$ is given by the Eq. (36) in Ref. [11].

Another $O\left(\alpha_{s}\right)$ contribution to the PNC comes from $t_{1}$-dependence of the LO HSC. As it is shown in Ref. [11], the LO factorization formula for $F_{2 q}\left(x_{B}, Q^{2}\right)$ in PRA can be rewritten as:

$$
F_{2 q}^{(L O P R A)}\left(x_{B}, Q^{2}\right)=e_{q}^{2} x_{B} f_{q}\left(x_{B}, Q^{2}\right)+\Delta F_{2 q}^{(f)}\left(x_{B}, Q^{2}\right)+\Delta F_{2 q}^{(T)}\left(x_{B}, Q^{2}\right)+\text { c.c. }+O\left(\left(Q^{2}\right)^{-\gamma}\right),
$$

where the first term is usual LO CPM result, "c.c." denotes the similar contribution of antiquark PDF and

$$
\begin{array}{r}
\Delta F_{2 q}^{(f)}\left(x_{B}, Q^{2}\right)=\int_{0}^{1} d x_{1} \int_{0}^{Q^{2}} d t_{1} T_{q}\left(t_{1}, Q^{2}, x_{1}\right)\left[f_{q}\left(x_{1}, Q^{2}\right)-f_{q}\left(x_{1}, t_{1}\right)\right] \cdot \frac{\partial}{\partial t_{1}} C_{2}^{(0)}\left(\frac{x_{B}}{x_{1}}, \frac{t_{1}}{Q^{2}}\right), \\
\Delta F_{2 q}^{(T)}\left(x_{B}, Q^{2}\right)=\int_{0}^{1} d x_{1} f_{q}\left(x_{1}, Q^{2}\right) \int_{0}^{Q^{2}} d t_{1}\left[1-T_{q}\left(t_{1}, Q^{2}, x_{1}\right)\right] \cdot \frac{\partial}{\partial t_{1}} C_{2}^{(0)}\left(\frac{x_{B}}{x_{1}}, \frac{t_{1}}{Q^{2}}\right),
\end{array}
$$

are non power-supressed corrections which arise because of the non-trivial dependence of the LO PRA HSC $C_{2}^{(0)}$ on $t_{1}$. Perturbative expansion of Eqns. (13) and (14) clearly starts from $O\left(\alpha_{s}\right)$, because both running of PDF with a scale and the difference of Sudakov formfactor from unity are preturbative effects. For the purpose of matching of the contribution (10) on the CPM result, only the term containing gluon PDF $f_{g}\left(x, Q^{2}\right)$ in $\Delta F_{2 q}^{(f)}$ is relevant and corresponding $O\left(\alpha_{s}\right)$ term can be computed using Eq. 13 and DGLAP equations:

$$
\Delta F_{2 q}^{\left(f_{g}\right)}\left(x_{B}, Q^{2}\right)=\frac{\alpha_{s}\left(Q^{2}\right)}{2 \pi}\left[\Delta C_{q g}^{\left(k_{T}\right)} \otimes f_{g}\right]
$$

where the coefficient $\Delta C_{q g}^{\left(k_{T}\right)}$ is written in Eq. (22) of Ref. [11].

In total, the $O\left(\alpha_{s}\right)$ contribution to the PNC for the subprocess (10) is given by

$$
F_{2 q}^{(1, g)}+\Delta F_{2 q}^{f_{g}}+\text { c.c. }=2 \frac{\alpha_{s}\left(Q^{2}\right)}{2 \pi}\left[f_{g} \otimes\left(C_{2 g}^{\overline{M S}}-\Delta C_{q g}+\Delta C_{q g}^{\left(k_{T}\right)}\right)\right]
$$

which is different from the NLO CPM result in $\overline{M S}$-scheme. In order to restore the agreement up to $O\left(\alpha_{s}\right)$, one has to compute the LO $\left(O\left(\alpha_{s}^{0}\right)\right)$ contribution not with the usual $\overline{M S}$ NLO PDFs, but with PDFs differing from them by some $O\left(\alpha_{s}\right)$ terms. These terms will cancel the contribution of $\Delta C_{q g}^{\left(k_{T}\right)}-\Delta C_{q g}$ in Eq. 15. Such special PDFs we call the PDFs in PRA scheme, see Eq. (40) of Ref. [11] for definition.

The reasoning above is needed only to determine explicitly the $\Delta C_{q g}^{\left(k_{T}\right)}$ and $\Delta C_{q g}$ contributions to scheme-transformation coefficient. In the complete NLO calculations, one should use NLO PRA unPDFs, generated from PDFs in PRA scheme with the help of Eqns. 12 and 13 in [11] and the contribution of subprocess (10) with corresponding mMRK subtractions is computed taking into account full $t_{1}$-dependence of the unsubtracted HSC and subtraction terms. When the full NLO calculation in PRA will be complete, and all contributions are taken together, the PNC should hold automatically, up to the terms $O\left(\alpha_{s}^{2}\right)$ and powersuppressed corrections.

The new features of loop calculations in Lipatov's EFT, such as rapidity divergences in loop integrals and Rapidity Renormalization Group are extensively reviewed in the Ref. [10]. To perform one-loop calculations in PRA a set of new one-loop integrals, containing lightcone propagators $1 / k^{ \pm}$and several scales of virtuality should be computed. 


\section{Acknowledgments}

The authors thank the Ministry of Education and Science of the Russian Federation for financial support in the framework of the Samara University Competitiveness Improvement Program among the world's leading research and educational centers for 2013-2020, the task number 3.5093.2017/8.9. The work of M.N. is supported in part by the Russian Foundation for Basic Research through the Grant No. 18-32-00060.

\section{References}

[1] B. L. Ioffe, V. S. Fadin, L. N. Lipatov, Quantum Chromodynamics perturbative and nonperturbative aspects, Cambridge University Press, 2010.

[2] J. C. Collins, Foundations of Perturbative QCD, Cambridge University Press, 2011.

[3] V. N. Gribov and L. N. Lipatov, Sov. J. Nucl. Phys. 15, 438 (1972); Yu. L. Dokshitzer, Sov. Phys. JETP 46, 641 (1977); G. Altarelli and G. Parisi, Nucl. Phys. B126, 298 (1977).

[4] J. Collins, Int. J. Mod. Phys. Conf. Ser. 37, 1560021 (2015).

[5] J. Collins and T. C. Rogers, Phys. Rev. D96, 054011 (2017).

[6] E. A. Kuraev, L. N. Lipatov, and V. S. Fadin, Sov. Phys. JETP 44, 443 (1976); I. I.

Balitsky and L. N. Lipatov, Sov. J. Nucl. Phys. 28, 822 (1978).

[7] L. N. Lipatov, and V. S. Fadin, Nucl.Phys. B406, 259 (1993).

[8] G.Watt, A.D.Martin, M.G.Ryskin, Eur.Phys.J. C31, 73 (2003)

[9] M.A. Nefedov, A.V. Karpishkov, V.A.Saleev, Phys. Rev. D96, 096019 (2017).

[10] M.A. Nefedov, and V.A. Saleev. Mod.Phys.Lett. A32, 1750207 (2017).

[11] M. Nefedov, V. Saleev, EPJ Web Conf. 158, 03011 (2017)

[12] L. N. Lipatov, Nucl. Phys. B452, 369 (1995).

[13] L. N. Lipatov and M. I. Vyazovsky, Nucl. Phys. B597, 399 (2001).

[14] L.N. Lipatov, Phys. Rept. 286, 131 (1997).

[15] T. C. Rogers, Phys. Rev. D 78, 074018 (2008)

[16] E. N. Antonov, L. N. Lipatov, E. A. Kuraev, and I. O. Cherednikov, Nucl. Phys. B721, 111 (2005).

[17] A. Buckley et al., Phys. Rept. 504, 145 (2011).

[18] M.A. Kimber, A.D. Martin and M.G. Ryskin, Phys. Rev. D63, 114027 (2001).

[19] M.A. Nefedov, V.A. Saleev and A.V. Shipilova, Phys. Rev. D87, 094030 (2013).

[20] V.A. Saleev and A.V. Shipilova, Phys. Rev. D86, 034032 (2012).

[21] R. Maciuła, V.A. Saleev, A.V. Shipilova and A. Szczurek, Phys. Lett. B758, 458 (2016).

[22] A.V. Karpishkov, V.A. Saleev and A.V. Shipilova, Phys. Rev. D94, 114012 (2016).

[23] A.V. Karpishkov, M.A. Nefedov, V.A. Saleev and A.V. Shipilova, Int. J. Mod. Phys. A30, 1550023 (2015).

[24] M.A. Nefedov and V.A.Saleev, Phys. Rev. D92, 094033 (2015).

[25] B.A. Kniehl, M.A. Nefedov and V.A. Saleev, Phys. Rev. D89, 114016 (2014).

[26] G. Aad et al. [ATLAS Collaboration], JHEP 1301, 086 (2013).

[27] G. Aad et al. [ ATLAS Collaboration], Phys. Rev. Lett. 106, 172002 (2011).

[28] T. Aaltonen et al. [CDF Collaboration], Phys. Rev. D84, 052006 (2011).

[29] V. Khachatryan et al. [CMS Collaboration], JHEP 1103, 136 (2011).

[30] T. Binoth, J. P. Guillet, E. Pilon and M. Werlen, Eur. Phys. J. C16, 311 (2000). 